\title{
3D scattering of waves by a cylindrical irregular cavity of infinite length in a homogeneous elastic medium
}

\author{
António J.B. Tadeu ${ }^{\mathrm{a}, *}$, Julieta M.P. António ${ }^{\mathrm{a}}$, Eduardo Kausel ${ }^{\mathrm{b}}$ \\ a Faculty of Sciences and Technology, Department of Civil Engineering, Polo II Pinhal de Marrocos, University of Coimbra, \\ 3030-290 Coimbra, Portugal \\ ${ }^{\mathrm{b}}$ Department of Civil and Environmental Engineering, Massachusetts Institute of Technology, Cambridge, MA 02139, USA
}

Received 17 July 2001; received in revised form 16 November 2001; accepted 21 November 2001

\begin{abstract}
The three-dimensional (3D) wave field scattered by an irregular, cylindrical cavity of infinite length contained in a homogeneous elastic medium illuminated by a dilatational point load is obtained. This model is used to evaluate the effect of the cross-sectional geometry of the cavity on the waves propagating in its vicinity. It particularly highlights the identification of the normal modes excited both in the frequency and time domain. The solution is formulated using the boundary element method for a wide range of frequencies and spatially harmonic line loads, which are then synthesized to obtain the time responses. The 3D solution is obtained as a summation of two-dimensional responses for different axial wavenumbers.

The responses in the frequency vs. axial-wavenumber domains are presented, allowing the recognition, identification, and physical interpretation of the variation of the wave field when five irregular cross-sections are used, namely a circle, an oval, a thin oval, a kidney and a boomerang. (c) 2002 Published by Elsevier Science B.V.
\end{abstract}

Keywords: Scattering of waves; Wave diffraction; Cavities in elastic media; Soil-structure interaction; Boundary element method; Elastodynamics; Seismology

\section{Introduction}

Measuring displacements in the vicinity of cavities, generated by a source either on the ground surface or buried, is an essential part of several geophysical and seismic prospecting techniques. The interpretation of the signals recorded during the course of some seismic testing techniques requires a full understanding of how the waves propagate from the source to the receiver. Depending on the position of the receivers and sources, and on the type of geological formation, it is known that very complex wave patterns may arise. A variety of numerical methods have been used to find the solution of wave propagation in cylindrical cavities.

\footnotetext{
${ }^{*}$ Corresponding author. Tel.: +351-239-797-100; fax: +351-239-797-123.

E-mail address: tadeu@dec.us.pt (A.J.B. Tadeu).
} 
Some of the first analytical studies on wave diffraction and scattering addressed the problems of wave motion and reverberations in alluvial basins of regular shape [1,2] and wave scattering elicited by cavities [3-6]. Liu and $\mathrm{Qu}[7,8]$ computed the dispersion equation for guided circumferential waves and analyzed the propagation of transient waves of this type in a hollow cylinder. Valle et al. [9] examined the propagation of time harmonic circumferential waves in a two-dimensional (2D), infinitely long hollow cylinder with an inner shaft, using analytical solutions written in a cylindrical coordinate system. The interfacial shear stresses were assumed to be zero, and the normal stress and radial displacements to be continuous. The dispersion curves of the first modes were presented. Semi-analytical methods have been used to analyze wave diffraction caused by geological irregularities of arbitrary shape within globally homogeneous media [10-12]. By contrast, the application of purely numerical methods (i.e. finite elements or finite differences combined with transmitting boundaries) has largely been restricted to situations where the response is required only within localized irregular domains, such as soil-structure interaction problems [13-15]. Discrete methods have also occasionally been used to model large alluvial basins, but only in plane-strain [16]. Manolis and Beskos [17] used the boundary element method (BEM) formulated in the Laplace transformed domain to compute the dynamic stress concentration in the lining of a circular tunnel within a linear elastic or viscoelastic medium of infinite extent under conditions of plane strain. Tsinopoulos et al. [18] described a BEM formulation combined with the fast Fourier transform to analyze general axisymmetric problems in frequency domain elastodynamics. Finally, hybrid methods involving a combination of finite elements, for modeling the interior domain containing the inhomogeneities, and semi-analytical representations, for the exterior domain, have been used as well [19].

The application of these numerical methods has been restricted mostly to situations where the solution is required within $2 \mathrm{D}$ domains. The reason is that the evaluation of the full scattering wave field generated by sources placed in the presence of three-dimensional (3D) propagation media requires the use of computationally demanding numerical schemes.

The solution becomes much simpler if the medium is $2 \mathrm{D}$, even if the dynamic source remains $3 \mathrm{D}$. Such a situation is frequently referred to as a two-and-a-half-dimensional problem (or $2 \frac{1}{2}$-D for short), for which solutions can be obtained by means of a spatial Fourier transform in the direction in which the geometry does not vary ( $z$ direction). This Fourier transform can be made discrete if it is assumed that the load is repeated at equal distances along the $z$ direction. Thus the solution requires first the calculation of a sequence of $2 \mathrm{D}$ problems with different spatial wavenumbers, $k_{z}$. Then, using the inverse Fourier transform, the $3 \mathrm{D}$ field is synthesized.

The solution of a $2 \frac{1}{2}$-D problem is known in closed form for inclusions with simple geometry, such as a circular cylinder, for which the wave equation is separable. If the inclusion has an irregular cross-section, however, the solution is more elaborate. In this latter case the BEM is perhaps the method of choice, particularly if the inclusion is buried in an unbounded elastic space or in elastic half-space, because of the ease with which to the far field conditions can be satisfied. Pedersen et al. [20] used the indirect boundary element method (IBEM) to study the 3D seismic response of 2D topographies to plane waves, making use of the full-space Green's functions for a harmonic point force moving along a direction parallel to the generatrix of the topography. Luco and De Barros [21,22] obtained the 3D harmonic response of an infinitely long shell with a circular cross-section, embedded in a layered viscoelastic half-space and subjected to harmonic plane waves impinging at an oblique angle relative to the axis of the shell. The technique used combines an indirect representation for the field in the exterior half-space and a model of the pipeline based on Donnel's shell theory. The integral representation for the soil is based on the use of moving Green's functions for the layered half-space. Stamos and Beskos [23] used the BEM to evaluate the 3D dynamic response of long, lined tunnels of uniform cross-section in a half-space when illuminated by plane harmonic waves propagating in an arbitrary direction. The problem was reduced to a $2 \mathrm{D}$ one by a coordinate transformation and appropriate integration of the full space dynamic fundamental solution along the direction of the tunnel axis. Other studies related to $2 \frac{1}{2}-\mathrm{D}$ are those of Zhang and Chopra [24,25], who used 
the direct boundary element method to determine the impedance matrix for a 3D foundation supported on an infinitely long canyon of uniform cross-section cut in a homogeneous half space.

In this paper, we look at the effect of non-circular boreholes, and compute the wavefield and motions elicited by monopole sources. Non-circular borehole cross-sections may occur for a variety of reasons, e.g, through the mechanical action of the drill string in vertically deviated wells, rock failure adjacent to a drilled borehole, or plastic deformation and washing out of the borehole in soft or poorly consolidated rocks [26,27]. Most of the published work has assumed the borehole to be circular, while there is paucity of research on non-circular boreholes. Burden [28] calculated and presented dispersion curves for the modes of elliptical boreholes in homogeneous elastic formations using a surface integral formulation that, after the surface displacements were approximated by a finite number of wave functions, was cast as a matrix equation. Bamberger et al. [29] studied the propagation of elastic waves along a cylindrical cavity, formulating the problem as an eigenvalue problem in the context of an unbounded self-adjoint operator. Their work involves a true mathematical approach where the existence of a hierarchy of guided modes, the existence of waves propagating for any value of wavenumber, and the presence of starting bands (i.e. dispersion branches for modes that exist only above some cut-off frequency) were demonstrated.

In this work, the BEM is used to evaluate the wave-field produced by a dilatational point load (blast load) in the vicinity of irregular cylindrical inclusions contained in an unbounded elastic medium: an oval, a thin oval, a kidney and a boomerang-shaped borehole cavity. The solution at each frequency is expressed in terms of waves with varying wavenumber $k_{z}$, which are then used to obtain time series by means of (fast) inverse Fourier transforms into space-time. The wavenumber transform in discrete form is obtained by considering an infinite number of virtual point sources equally spaced along the $z$ axis and at a sufficient distance from each other to avoid spatial contamination [30]. In addition, the analyses are performed using complex frequencies, shifting down the frequency axis in the complex plane, in order to minimize the influence of the neighboring fictitious sources [31].

This paper is organized as follows: first, the problem is defined and the BEM formulated; then, the method is implemented and validated by applying it to a circular cylindrical cavity buried in an infinite homogeneous elastic medium, which is subjected to a point dilatational load for which the solution is calculated in closed form; finally, the BEM is used to evaluate the wave-field elicited by a point dilatational load in the presence of an irregular cylindrical cavity in an unbounded elastic medium. Simulation analyses with this idealized model are used to study the patterns of wave propagation in the vicinity of these inclusions when different cylindrical cross-sections of equal perimeter are used. The amplitude of the wavefield in the frequency vs. axial-wavenumber domain is presented, allowing the recognition, identification, and physical interpretation of the variation of the wave field.

\section{Problem definition}

Consider an irregular cylindrical cavity of infinite extent, buried in a spatially uniform elastic medium (Fig. 1), subjected to a harmonic dilatational point source at position $(0,0,0)$, pulsating with a frequency $\omega$. The incident field can be expressed by means of the classical dilatational potential $\phi$

$$
\phi_{\text {inc }}=\frac{A \exp \left[i \frac{\omega}{\alpha}\left(\alpha t-\sqrt{x^{2}+y^{2}+z^{2}}\right)\right]}{\sqrt{x^{2}+y^{2}+z^{2}}}
$$

in which the subscript inc denotes the incident field, $A$ is the wave amplitude, $\alpha$ is the compressional wave velocity of the medium, and $\mathrm{i}=\sqrt{-1}$. 


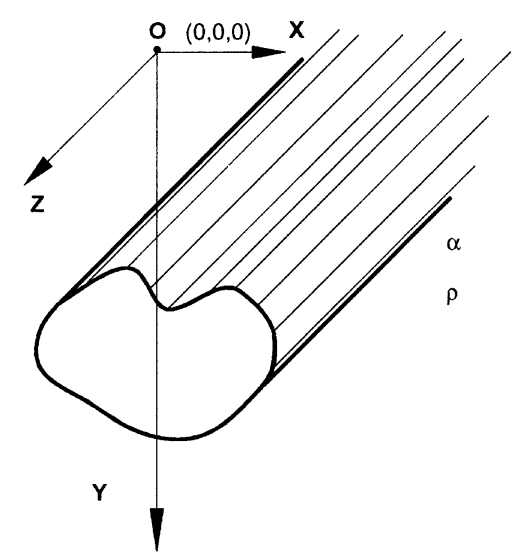

Fig. 1. Geometry of the problem.

Defining the effective wavenumbers

$$
k_{\alpha}=\sqrt{\frac{\omega^{2}}{\alpha^{2}}-k_{z}^{2}}, \quad \operatorname{Im} k_{\alpha} \leqslant 0
$$

by means of the axial wavenumber $k_{z}$, and Fourier-transforming Eq. (1) in the $z$ direction, one obtains

$$
\hat{\phi}_{\text {inc }}\left(\omega, x, y, k_{z}\right)=\frac{-\mathrm{i} A}{2} H_{0}^{(2)}\left(k_{\alpha} \sqrt{x^{2}+y^{2}}\right)
$$

in which the $H_{n}^{(2)}(\cdots)$ are second Hankel functions of order $n$.

If one considers an infinite number of virtual point sources equally spaced along the $z$ direction at a sufficient distance, $L$, from each other to avoid spatial contamination [30], the incident field may be written as

$$
\phi_{\text {inc }}(\omega, x, y, z)=\frac{2 \pi}{L} \sum_{m=-\infty}^{\infty} \hat{\phi}_{\text {inc }}\left(\omega, x, y, k_{z}\right) \mathrm{e}^{-\mathrm{i} k_{z m} z}
$$

with $k_{z m}=(2 \pi / L) m$. This equation converges and can be approximated by a finite sum of terms.

\section{Boundary element formulation}

The 3D field generated by a cylindrical cavity subjected to spatially sinusoidal harmonic line loads (defined by Eq. (3)) is found by means of the BEM. Since the basic equations used when applying boundary elements to wave propagation are widely known [32,33], it is enough to state that, for the method to be applied for the scattering problem presented herein, the following integral in the frequency domain must be evaluated:

$$
H_{i j}^{k l}=\int_{C_{l}} \phi H_{i l}\left(x_{k}, x_{\mathrm{s}}, n_{\mathrm{s}}\right) \mathrm{d} C_{l} \quad(i, j=1,2,3)
$$

where $H_{i j}\left(x_{k}, x_{\mathrm{s}}, n_{\mathrm{s}}\right)$ represents the traction components at the point $x_{k}$ in direction $i$ caused by a concentrated load acting at the source point $x_{\mathrm{s}}$ in direction $j, n_{\mathrm{s}}$ is the unit outward normal for the $l$ th boundary segment, and $\phi$ are the interpolation functions. 


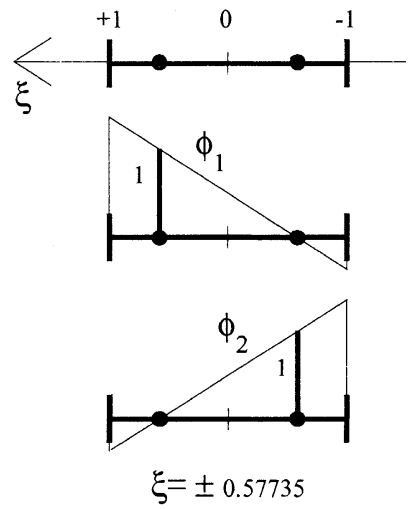

Fig. 2. Interpolation functions and position of the nodal points.

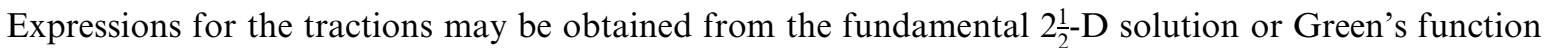
$G_{i j}$, by taking partial derivatives to deduce the strains and then applying Hooke's law to find the stresses. The displacement and stress functions that apply to the present case are listed in Appendix A.

The displacement and stress variations within a boundary element are defined in terms of the nodal values. Linear interpolation functions are used to map the nodal values, that is to say, boundary elements with two nodal points have been used. The traction discontinuity at the corner between two boundary elements is handled by moving the nodes, which would meet at the corner, so that each is inside an element [34] leading to discontinuous boundary elements. The nodal points are chosen to coincide with those in a Gauss-Legendre numerical integration (Fig. 2).

After mathematical manipulation of the integral equations, combined and subjected to the continuity conditions at the interface between the two media, and discretized appropriately, a system of equations that can be solved for the nodal displacements is obtained. The required integrations in Eq. (5) are performed using Gaussian quadrature, when the element to be integrated is not the loaded element, using eight Gaussian points. For the loaded element, on the other hand, the singular integrands are evaluated out in closed form $[35,36]$.

\section{Validation of the BEM algorithm}

The BEM algorithm was implemented and validated by applying it to a circular cylindrical cavity, placed in a homogeneous elastic medium $\left(\alpha=4208 \mathrm{~m} / \mathrm{s}, \beta=2656 \mathrm{~m} / \mathrm{s}\right.$ and $\left.\rho=2140 \mathrm{~kg} / \mathrm{m}^{3}\right)$. It was then subjected to a harmonic line dilatational load applied at point $\mathrm{O}$, with $k_{z}=1.0 \mathrm{rad} / \mathrm{m}$, as in Fig. 3a, for which the solution is known in closed form and described in Pao and Mow [37].

The response is calculated at receiver $R 1$, placed along a line parallel to the longitudinal axis of the cylinder $(x=0.0 \mathrm{~m}, y=-0.3 \mathrm{~m})$. The computations are performed in the frequency range $(31.25,4000$ $\mathrm{Hz}$ ), with a frequency increment of $31.25 \mathrm{~Hz}$. The spatial period considered in the analysis is $L=269 \mathrm{~m}$.

The number of elements used to model the cavity is defined by the ratio between the wavelength of the incident waves and the length of the boundary elements (set to be 15.0). The minimum number of elements is never less than 40. Fig. $3 \mathrm{~b}$ displays an example of a BEM mesh.

Fig. 3c displays the real and imaginary parts of the $z$ displacement. In this figure, the continuous line represents the analytical solution while the marked points represent the BEM solution. Analysis of the results confirms a good agreement between the two solutions. 

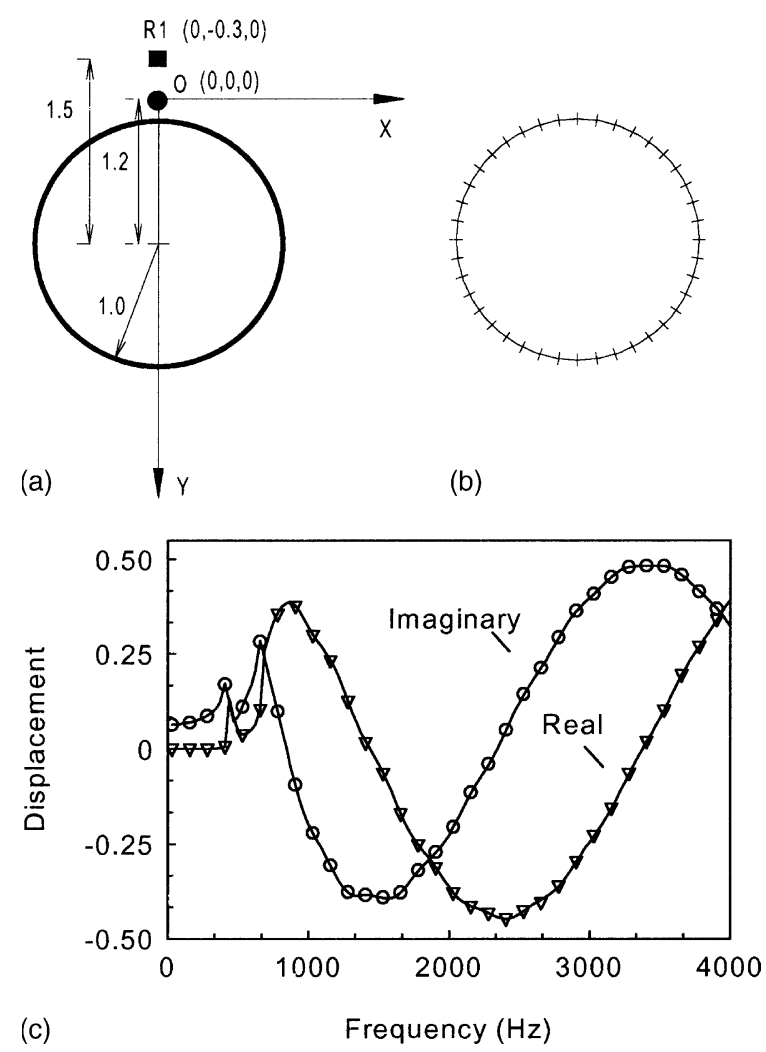

Fig. 3. Validation of the BEM: (a) circular cylindrical inclusion in an unbounded elastic medium, (b) typical mesh used (40 elements), (c) scattered field displacement at receiver \#1 in $z$ direction: analytical solution; numerical solution for $k_{z}=1.0 \mathrm{rad} / \mathrm{m}$ and $\lambda / L=15$.

\section{Results in space-time}

The displacements in the spatial-temporal domain are obtained by a numerical fast Fourier transform in $k_{z}$, considering a source whose temporal variation is given by a Ricker wavelet, as defined below. This wavelet form is chosen because it decays rapidly in both time and frequency, which not only reduces the computational effort, but also allows easier interpretation of the computed time series and synthetic waveforms.

The Ricker wavelet function is given by

$$
u(\tau)=A\left(1-2 \tau^{2}\right) \mathrm{e}^{-\tau^{2}}
$$

where $A$ is the amplitude, $\tau=\left(t-t_{\mathrm{s}}\right) / t_{0}$ and $t$ denotes time; $t_{\mathrm{s}}$ is the time when the maximum occurs, while $\pi t_{0}$ is the characteristic (dominant) period of the wavelet. Its Fourier transform is

$$
U(\omega)=A\left[2 \sqrt{\pi} t_{0} \mathrm{e}^{-\mathrm{i} \omega t_{\mathrm{s}}}\right] \Omega^{2} \mathrm{e}^{-\Omega^{2}}
$$

in which $\Omega=\omega t_{0} / 2$.

As stated before, the Fourier transformations are achieved by discrete summations over wavenumbers and frequencies, which is mathematically equivalent to adding periodic sources at spatial intervals $L=2 \pi / \Delta k_{z}$ (in the $z$-axis), and temporal intervals $T=2 \pi / \Delta \omega$, with $\Delta k_{z}$ and $\Delta \omega$ being the wavenumber and frequency steps, respectively [30]. The spatial separation $L$ is chosen sufficiently large to prevent contami- 
nation of the response by the periodic sources. In other words, the contribution to the response by the fictitious sources must be guaranteed to occur at times later than $T$. This goal can also be aided substantially by shifting the frequency axis slightly downward, that is, by considering complex frequencies with a small imaginary part of the form $\omega_{\mathrm{c}}=\omega-\mathrm{i} \eta$ (with $\eta=0.7 \Delta \omega$ ). This technique results in a significant attenuation or virtual elimination of the periodic sources. In the time domain, this shift is later taken into account by applying an exponential window $\mathrm{e}^{\eta t}$ to the response [38].

\section{Numerical applications}

Simulation analyses are performed for the case of a cylindrical cavity in an elastic unbounded medium that is illuminated by an incident blast load defined by the dilatational potential $\phi$ given by Eq. (1). Five inclusions with different cross-sections are considered, namely a circle, an oval with an aspect ratio $\varepsilon=1.20 / 0.76=1.58$, a thin oval, with an aspect ratio $\varepsilon=1.4 / 0.52=2.69$, a kidney and a boomerang. The kidney and the boomerang are obtained from the oval and thin oval inclusion, respectively, by deforming the side wall. The perimeter of all cavities is constant $(2 \pi \mathrm{m})$.

At time $t=0$, a point source at a point $\mathrm{O}$, placed $0.2 \mathrm{~m}$ from the surface of the inclusion, creates a spherical dilatational pulse propagating away from $\mathrm{O}$. The field generated is computed at receivers located on five planes, equally spaced $(10.0 \mathrm{~m})$ along the $z$ direction, placed around the inclusion, $0.5 \mathrm{~m}$ (\#1 and \#3) or $3.0 \mathrm{~m}(\# 2)$ from its surface, as shown in Fig. 4.

The dilatational wave velocity $(\alpha=4208 \mathrm{~m} / \mathrm{s})$, the shear wave velocity $(\beta=2656 \mathrm{~m} / \mathrm{s})$ and density $\left(\rho=2140 \mathrm{~kg} / \mathrm{m}^{3}\right)$ of the host elastic medium are kept constant for all analyses. The computations are performed in the frequency range $[31.25,4000 \mathrm{~Hz}$, with a frequency increment of $31.25 \mathrm{~Hz}$, which results in a total time of $(T=32.0 \mathrm{~ms})$ for the analysis in the time domain. This in turn implies that the spatial period cannot be less than $L=2 T \alpha=269 \mathrm{~m}$. We choose $\eta=0.7 \Delta \omega$ as the imaginary part of the angular frequency, so as to attenuate the wraparound by a factor of $\mathrm{e}^{0.7 \Delta \omega T}=81$ (i.e., $38 \mathrm{~dB}$ ). The source time dependence is a Ricker wavelet with a characteristic frequency of $1500 \mathrm{~Hz}$.

The cavities are modeled with boundary elements, the number of which changes with the excitation frequency of the harmonic load. A ratio between the wavelengths of the incident waves and the length of the boundary elements is kept at a minimum of 15.0. In all cases the minimum number of the boundary elements used to model each cavity is at least 40 .

Of the various waves propagating along the boundary of the cylinder, two are non-dispersive (body waves), namely the dilatational (P) and shear (S) waves. The time records show the existence of an incident $\mathrm{P}$ pulse and a $\mathrm{P}$ pulse reflected from the surface of the inclusion ( $\mathrm{P}$ and $\mathrm{PP}$, respectively, in seismological nomenclature); secondary arrivals are $\mathrm{S}$ waves that are converted from a $\mathrm{P}$ wave impinging onto the surface (PS). Fig. 5 shows the propagation path of these pulses in the $z$ direction between the source and the receivers \#2, located at $z=20.0$ and $60.0 \mathrm{~m}$ from the plane source, when a circular inclusion is present. Tags are added in these plots, indicating the arrivals of the various pulses. The arrival times obtained for the $\mathrm{P}$, PP and PS pulses in these plots are consistent with the predictions given by ray acoustics. In addition, there are various types of guided waves - the normal modes - propagating along the surface of the cavity at a slower velocity than the shear (S) waves. Axisymmetric modes and modes with azimuth variation are also excited. Some of these modes are excited only if the source excitation frequency exceeds the cutoff (or resonant) frequencies of the cylinder. Ewing et al. [39] has indicated that these wave types do not satisfy a minimum time condition, and thus do not satisfy the law of geometric acoustics.

The dispersion characteristics of the normal modes can be obtained by solving an eigenvalue problem for free waves that propagate in the absence of external sources. The associated eigenvalues $k_{z}$, lead in turn to the phase and group velocities of the normal modes. Although an infinite (but countable) number of modes exist, only those with low modal order contribute significantly to the response. 

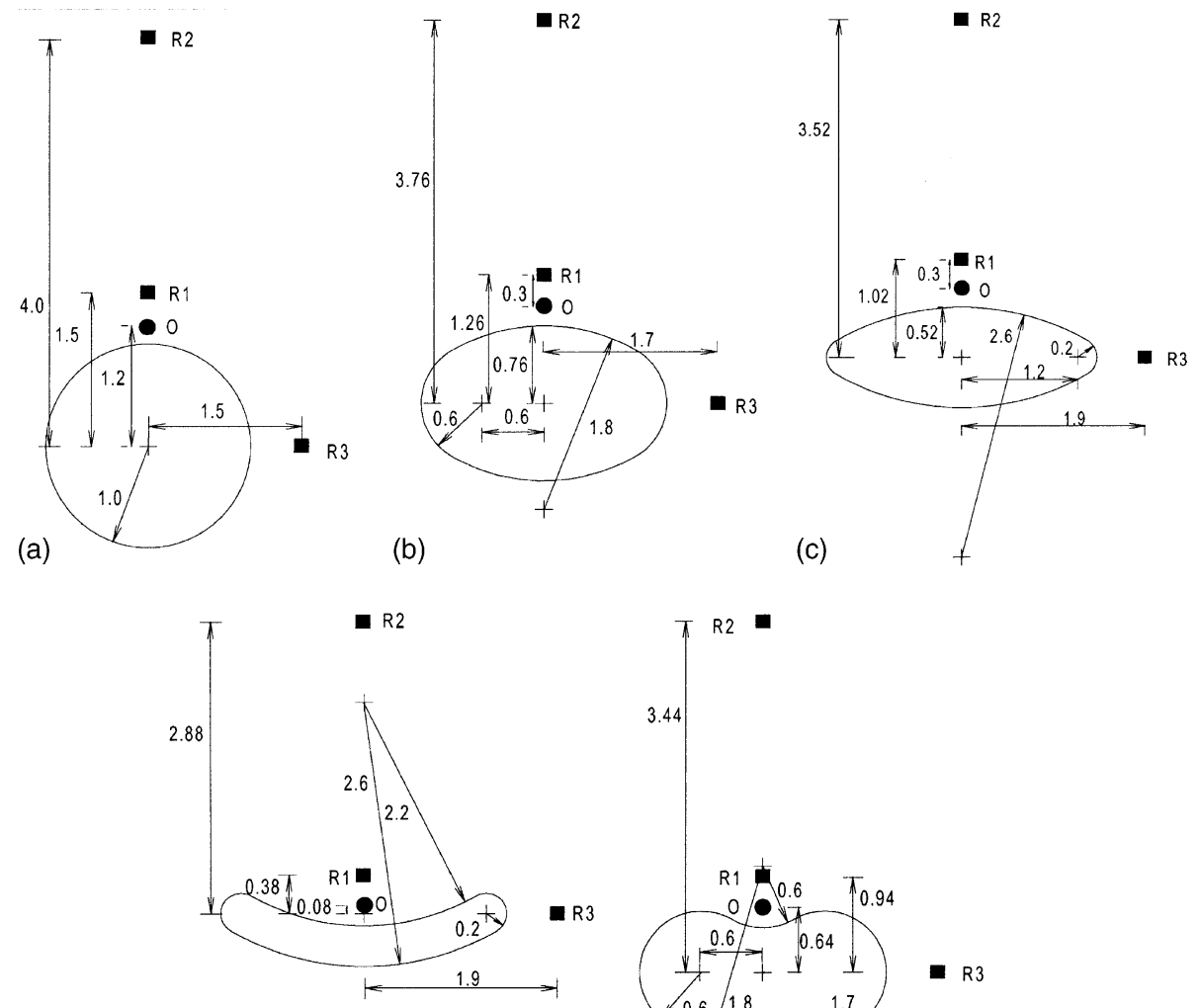

(d)

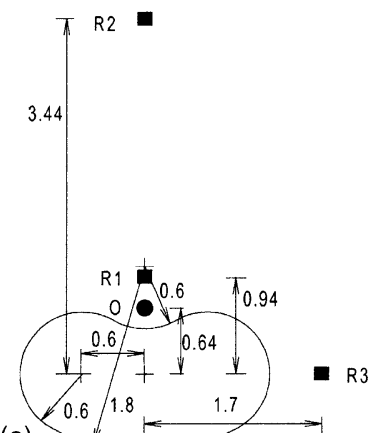

(e)

Fig. 4. Cross-sections of the fixed cylindrical irregular cavities: (a) circular; (b) oval; (c) thin oval; (d) boomerang; (e) kidney.

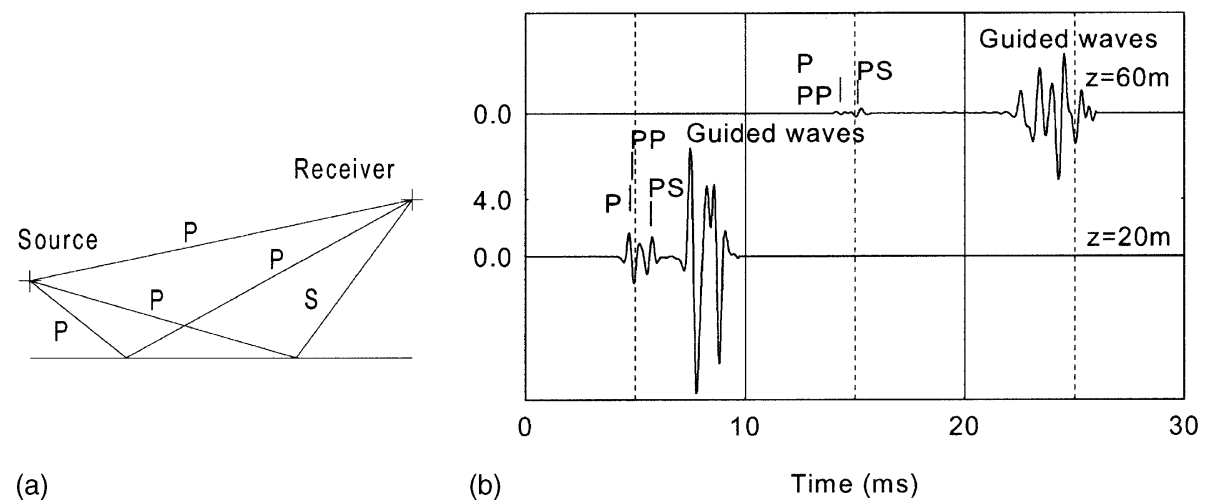

Fig. 5. Flight time of different body pulses travelling between the source $\mathrm{S}$ and the receiver \#2, when a circular inclusion is presented: (a) propagation path scheme of the different pulses, (b) time responses for receivers located at $z=20.0 \mathrm{~m}$ and $z=60.0 \mathrm{~m}$ from the plane source.

Next, selected results are used to illustrate the main findings. In addition to the time responses, Fourier spectra results are included since different wave types in the frequency vs. axial-wave number domain are 

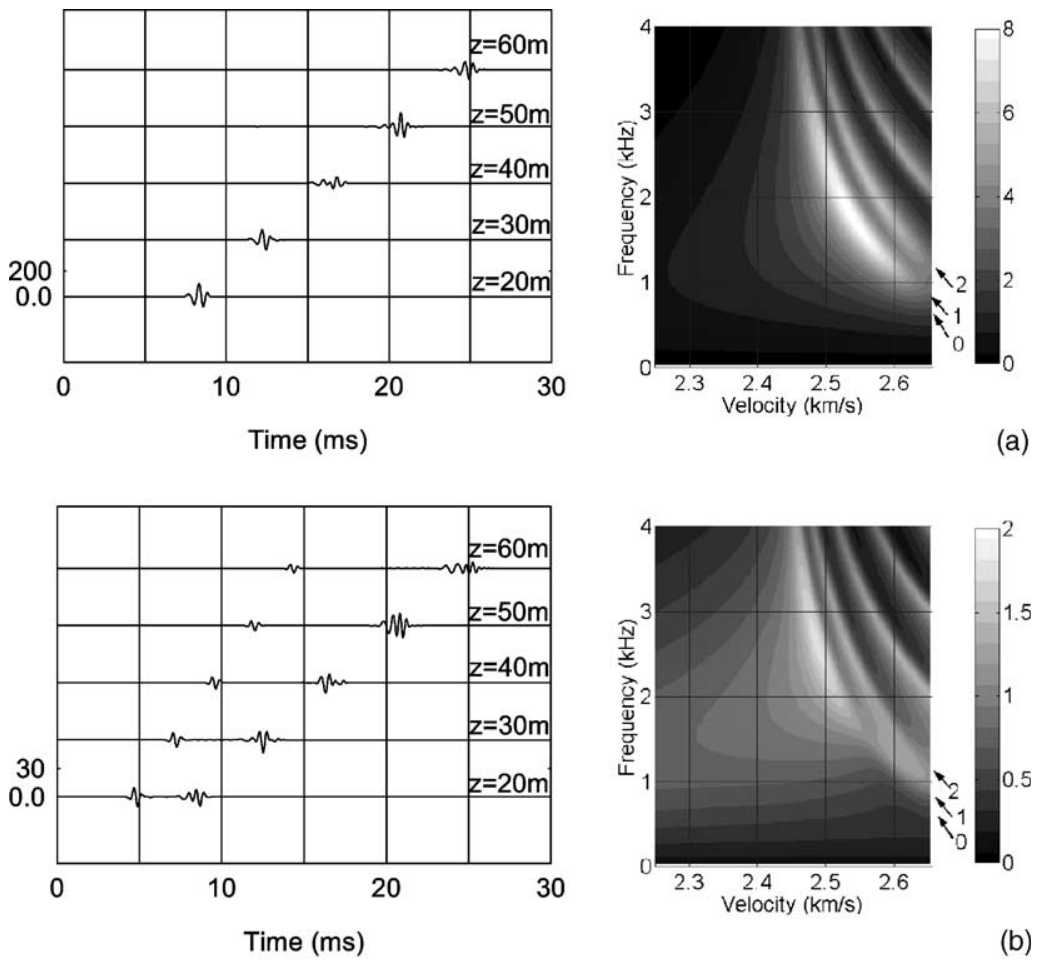

Fig. 6. Time and Fourier spectra responses at receivers \#1 in the presence of a circular cylindrical cavity: (a) vertical displacement; (b) $z$ displacement.

more easily recognized and identified, as they occupy specific sub-domains, according to their phase wave velocity. As the response is dominated by the behavior of the guided waves, the displayed Fourier spectra are zoomed for waves with slower phase velocity than the shear wave. These plots use a gray scale where light and dark shades are ascribed to high and low amplitude values, respectively.

Figs. 6 and 7 display the responses of a circular inclusion at receivers \#1 and \#3, respectively (see Fig. 4a). The horizontal displacements at receivers \#1, placed along the vertical $y$ axis, are null, given that this axis constitutes an axis of symmetry (absent in Fig. 6). The vertical displacements at these receivers do not show the arrival of the body waves (P, PP and PS) clearly, because their amplitude is tiny compared with the amplitude of the guided waves. Meanwhile, the $z$ responses exhibit the arrival of the body waves, but they do not fully separate into different sets of pulses, because the characteristic frequency of the excitation of the source does not lead to pulses with a time duration sufficiently small to avoid their overlapping. The existence of guided waves is clearly visible, and these correspond to waves travelling at slower velocities than the shear wave. The guided waves are originated on the surface of the cavity and they travel along its surface. Their amplitude decays as the distance to the cavity surface increases and they exhibit larger amplitudes along the normal direction of the cavity, and so the body waves are visible for $z$ displacements because of the smaller amplitude of the guided waves. The Fourier spectra show the existence of different mode types. In these figures, the first order modes are identified by a number, indicating the variation of the mode with the azimuth. The first axisymmetric mode is the pseudo-Rayleigh wave (0) which is associated with slow wave velocities. It shows a cutoff frequency at around $0.9 \mathrm{kHz}$, where the mode reaches its highest phase velocity, namely, the shear wave velocity of the solid. As wave frequency increases, the phase velocity 

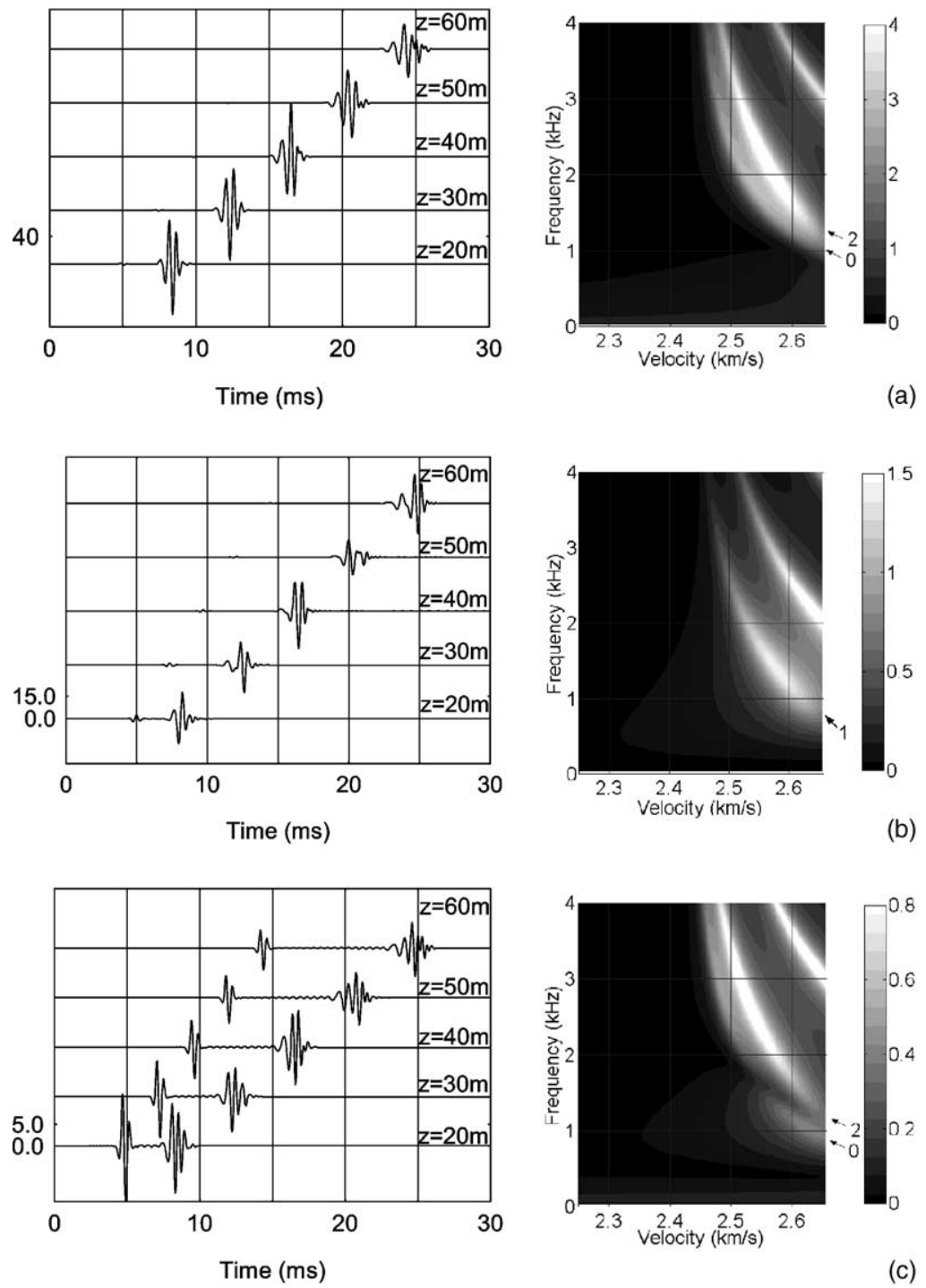

Fig. 7. Time and Fourier spectra responses at receivers \#3 in the presence of a circular cylindrical cavity: (a) horizontal displacement; (b) vertical displacement; (c) $z$ displacement.

of these waves decreases and approaches the speed of the Rayleigh waves in a half space [29]. An analysis of the response allows us to verify that this first mode is highly dispersive. The first mode with azimuth variation is the flexural mode (1) while the third mode corresponds to the screw mode (2). Other, higher order, modes occur after these modes. The motion normal to the cavity surface of the first axisymmetric mode, a pseudo-Rayleigh wave, is essentially a deformed dilation, while the flexural mode is essentially a localized translation in the $y$ direction. The axisymmetric mode generates null vertical displacements at receivers \#3, while the flexural mode produces null horizontal and $z$ displacements there (see Fig. 7), because this receiver is placed on a neutral axis ( $x$ axis). The screw mode also generates null vertical displacements at this receiver. 
As observed in the time responses, the Fourier spectra plots exhibit higher amplitudes along the normal directions to the cavity. However, there is a marked drop in the amplitude of guided waves as we move from receiver \#1 to \#3, as a result of the increased energy dispersion. Notice that receivers \#1 are placed close to the source, while receivers \#3 are further away from the source and in a different azimuth distance (see Fig. 4a). Meanwhile, at the receivers \#3, the first axisymmetric mode waves lose importance in comparison with the other, higher order, wave guided modes.

Fig. 8 displays the vertical time and Fourier spectra responses at receiver \#1, placed $0.5 \mathrm{~m}$ above the inclusion, obtained for the four different irregular cross-sections of inclusions analyzed in this work. As we move from the circular (Fig. 6a) to the oval $\varepsilon=1.58$ (Fig. 8a), and then to the thin oval $\varepsilon=2.69$ (Fig. 8b), the spectra plots exhibit the existence of axisymmetric and flexural mode waves with progressively slower velocity. The time plots confirm this phenomenon by placing the guided pulses with a progressively delayed arrival. It can be further observed that the amplitude of the guided waves increases as the surface inclusion becomes flatter (greater curvature radius). This phenomenon may be explained by the reflective power of the convex shape of the cavity, which enables energy to be reflected to the sides, away from the top surface. As the radius of the curvature of the inclusion increases, its convexity diminishes and leads to a smaller energy spread to the sides of the inclusion. This behavior is even more pronounced as we move from a thin oval to the boomerang (Fig. 8c), whose shape enables the energy to be concentrated in the concave part of the inclusion. The slower wave modes further reduce their velocity. The concentration of energy at the receivers \#1 is even more pronounced in the case of the kidney shaped inclusion (Fig. 8d), because of the narrower concavity. Meanwhile the velocity of the slower guided wave increases, apparently as a result of the decrease of the curvature radius of the concave part of the inclusion.

As the upper surface of the inclusion gets flatter, the pseudo-Rayleigh wave velocity (axisymmetric mode waves) decreases and it appears that it approaches the Rayleigh wave velocity in a half-space $\left(\alpha_{R}=2407 \mathrm{~m} / \mathrm{s}\right)$ (see Fig. 8a-d). It can further be observed that these waves show the existence of a cutoff frequency, which increases slightly as the upper surface of the inclusion gets flatter. The velocity of this mode shows higher dispersion at low frequencies, which tends to increase as we move from the circular to the boomerang cross-section. The amplitude of these waves grows as the upper surface gets flatter (see Fig. 8a and b), and then when the concavity of the upper surface gets narrower (see Fig. 8c and d).

The flexural waves exist for all frequencies. When the cavity is circular (Fig. 6a) the flexural mode waves undergo a dispersion behavior similar to that found for the axisymmetric mode. As the elliptical aspect ratio $(\varepsilon)$ grows, at lower frequencies, the dispersion curves of these two modes appear to have distinct behavior, while at higher frequencies their wave phase velocities are closer. This effect agrees with the tendency reported by Burden [28] in the calculation of the dispersion curves for surface waves along cylindrical cavities of general cross-section.

As we move from the circular inclusion to the oval, the cutoff frequency of the screw mode increases, the mode becomes less dispersive and exhibits lower amplitude. Moving on to the thin oval, this same trend is maintained, with the importance of the screw mode continuing to decline. (Fig. $8 \mathrm{~b}$ does not illustrate a clear screw wave mode because it overlaps a higher mode). In addition, as we move from the oval to the kidney cross-section, the importance of the higher order modes also decreases, and a very weak contribution of the screw mode is registered.

As observed for the circular cross-section, the $z$ displacements at receivers \#1 for all other cross-sections clearly exhibit the arrival of the body waves. These first arrivals are not significantly different when we move from the circular cross-section to the other shapes. By contrast, the guided waves show features very similar to those found for the vertical responses. Fig. 9 displays, as an example, the time responses obtained for the oval and kidney. As observed before, the guided waves are enhanced when we move from the oval to the kidney shape.

The horizontal displacement at the receivers \#3 denotes weaker amplitude for the guided waves as the ovality ratio increases. The results show a further amplitude decrease when the side wall of the cavity is 

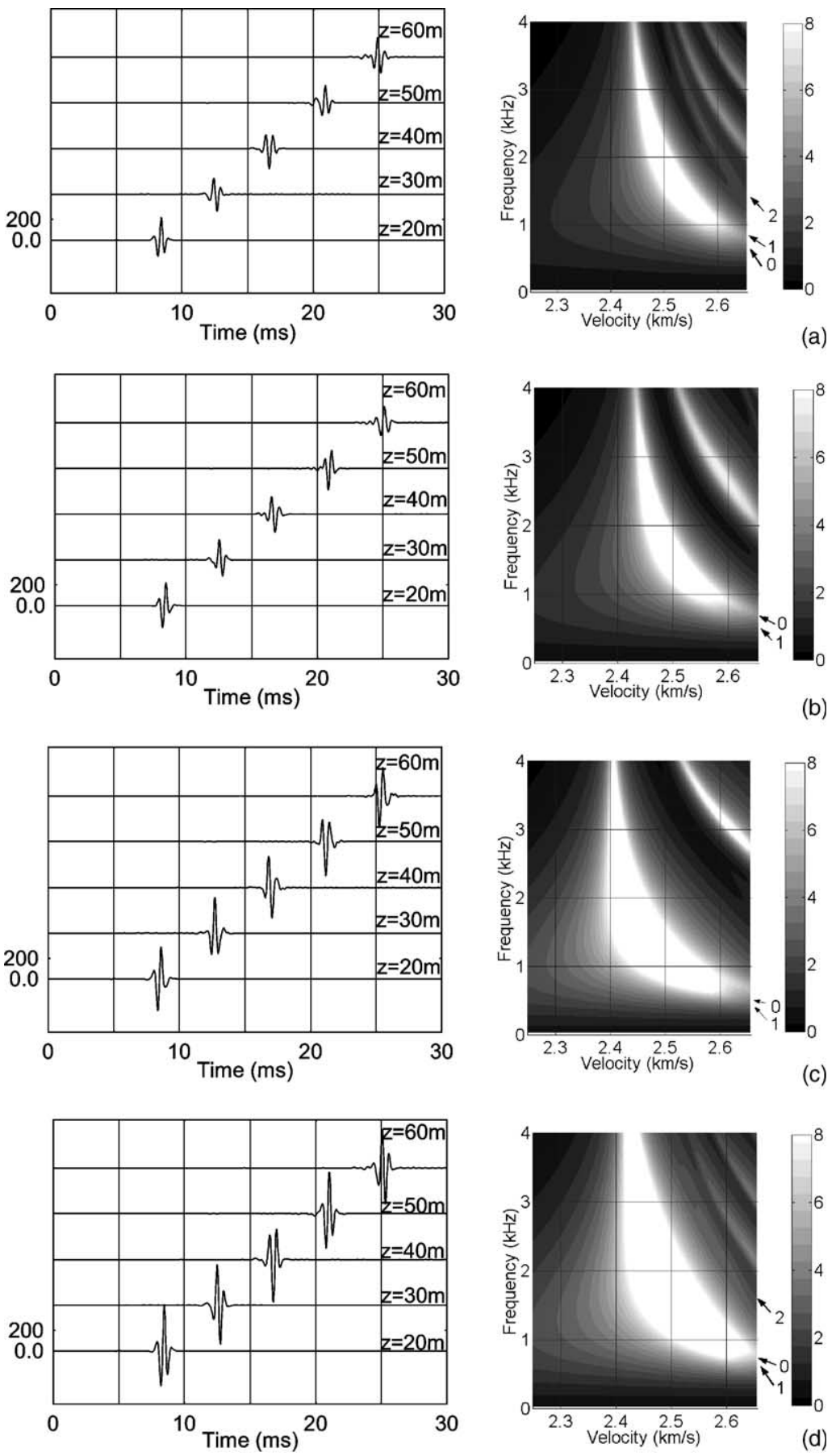

Fig. 8. Time and Fourier spectra vertical responses at receivers \#1: (a) oval; (b) thin oval; (c) boomerang; (d) kidney. 


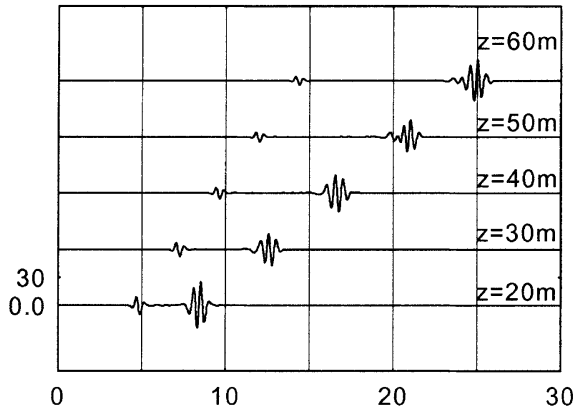

(a)

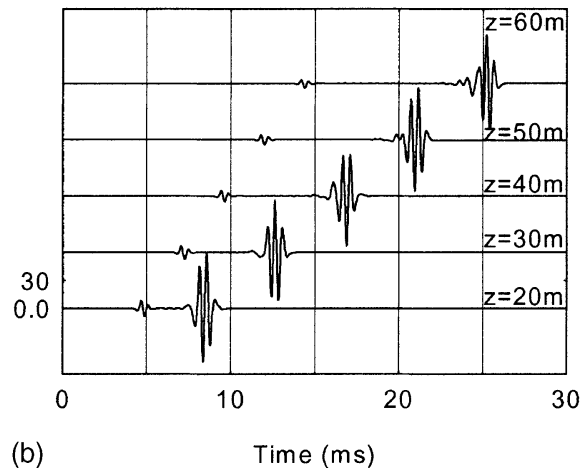

(b)

Fig. 9. Time responses at receivers \#1 in direction $z$ : (a) oval; (b) kidney.

deformed from an oval into a kidney, and from a thin oval into a boomerang. This phenomenon can be explained by the fact that more energy is retained above the cavity, as the upper surface of the cavity gets flatter and then concave, as described previously. Fig. 10 illustrates this behavior by displaying the horizontal time responses for the oval and the kidney. Notice, that the $x$ axis is not a neutral axis when the cross-section is a kidney-shaped cavity. Indeed, the spectra responses show the existence of weak flexural waves, which are absent when the cavity is an oval.

The vertical displacements at this same receiver only include contributions from the flexural waves when the cavity is regular. However, when the cavity is irregularly deformed (a kidney- or a boomerang-shaped
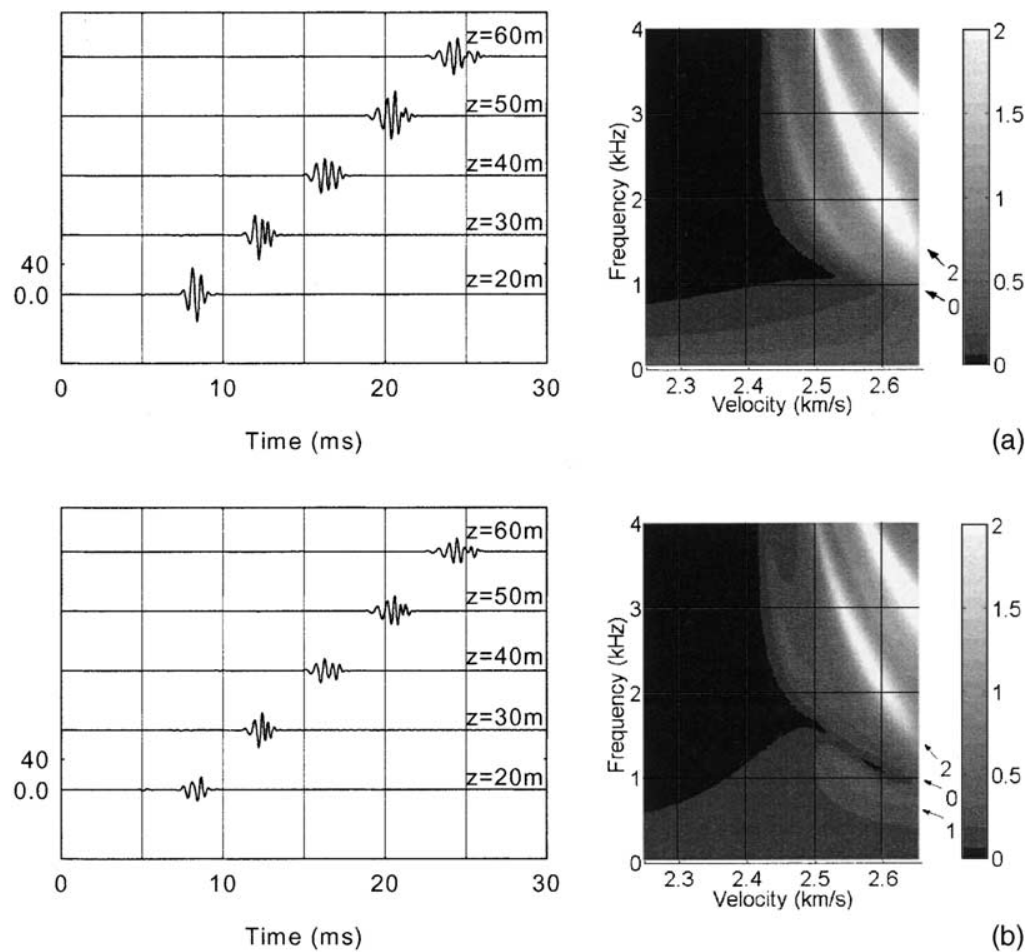

Fig. 10. Time and Fourier spectra horizontal responses at receivers \#3: (a) oval; (b) kidney. 
inclusion) the results show the existence of other surface mode types, in addition to the flexural waves (not illustrated).

Fig. 11 illustrates the time displacements at the receivers \#3, along the $z$ direction. Analysis of these plots reveals the existence of similar amplitudes for the body and guided waves. The development of the body pulses with time is similar for the circular (Fig. 7c), the oval (Fig. 11a) and the thin oval cavities (Fig. 11b), while it reveals the existence of later arrivals when their side wall is deformed (boomerang-Fig. 11c and kidney-Fig. 11d). This phenomenon is more pronounced when the results are computed for the boomerang, which denotes the existence of higher reverberations within the concave part of the inclusion. The guided pulses' patterns are more complicated when the cavity is circular, becoming simpler as the ovality of the cross-section increases (see Fig. 11a and b). This is because some of the higher modes both lose importance and possess higher cutoff frequencies, as we move from the circular to the oval and then to the thin oval cross-section, confirming the description above. It can be further observed that the guided pulses appear more complex as we move from the oval to the kidney, and from the thin oval to the boomerang, because other higher modes gain in importance.

The vertical displacement responses computed at receiver \#2 (Fig. 12), placed away from the cavity, show some of the features generated at receiver \#1. The guided waves decay significantly, but their contribution is still important. The body arrivals are now clearly visible, and they reveal differences related to the type of cross-section, becoming more pronounced when the top surface gets flatter and then concave. Again, the first modes (axisymmetric and flexural) become slower as the ovality of the cavity increases, and as the cavity is deformed to give a concave cavity. However, the amplitude of the responses, computed for the various crosssection cavities is similar. Furthermore, these first modes are only important at low excitation frequencies.

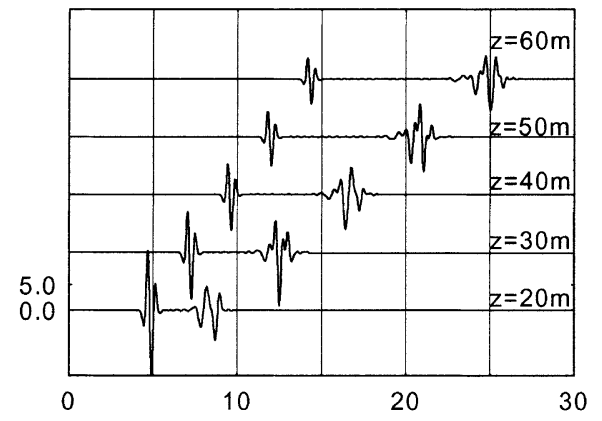

(a)

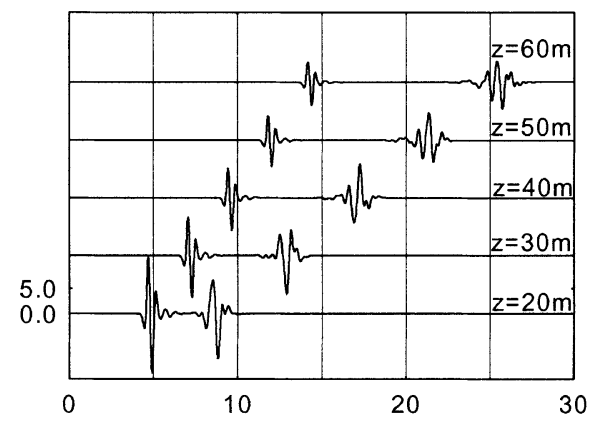

(c)

Time $(\mathrm{ms})$

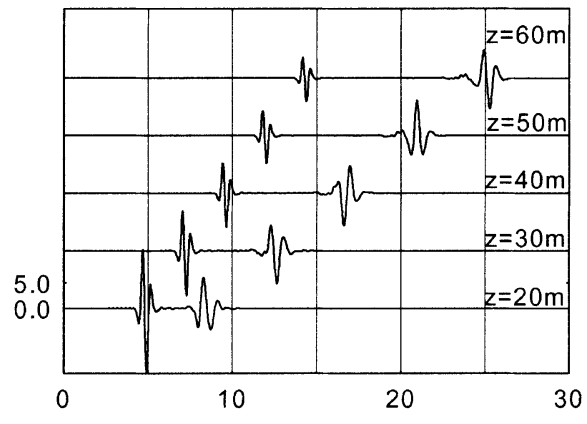

(b)

Time (ms)

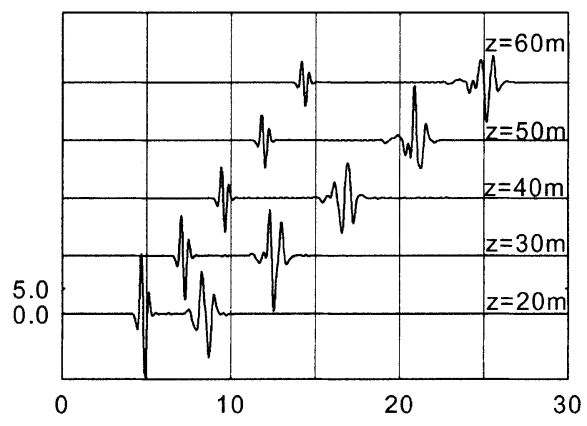

(d)

Time (ms)

Fig. 11. Time responses at receivers \#3 in direction $z$ : (a) oval; (b) thin oval; (c) boomerang; (d) kidney. 


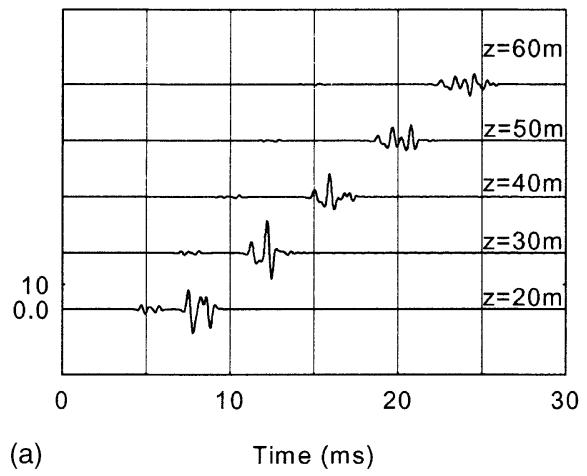

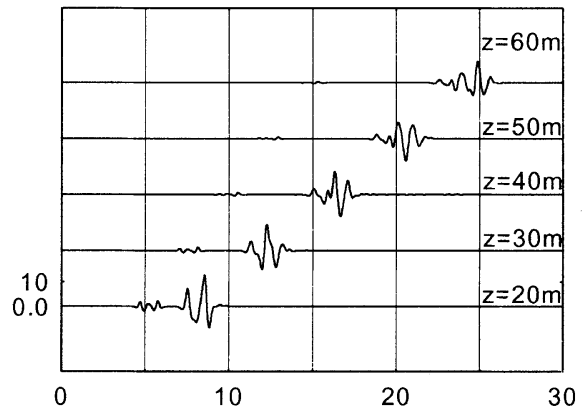

(b)

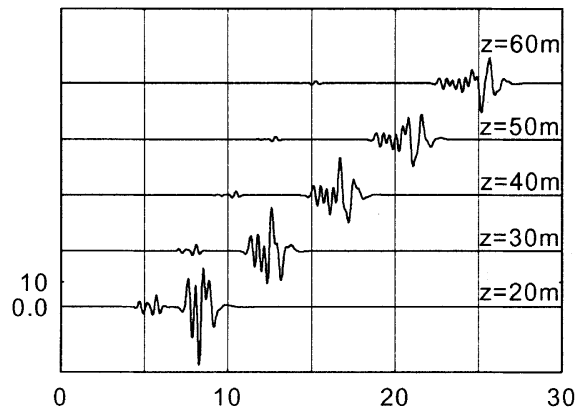

(d)
Time (ms)

Time (ms)

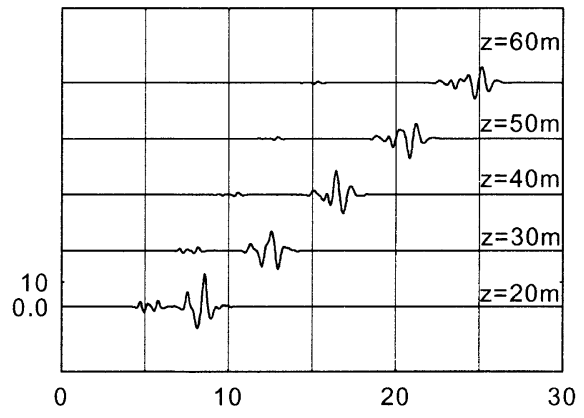

(c)

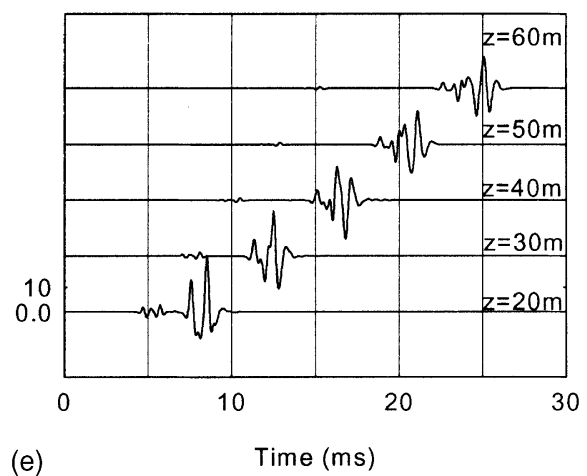

(e)

Fig. 12. Time vertical responses at receivers \#2: (a) circular; (b) oval; (c) thin oval; (d) boomerang; (e) kidney.

Again, the pattern of guided pulses increases in complexity as the cavity becomes concave, for which higher velocity modes now gain importance. It can even be observed that the time responses show the arrival of the first guided waves, with enhanced amplitudes.

\section{Conclusions}

A boundary element formulation was developed to assess the 3D scattered field generated by dilatational point loads illuminating non-circular cylindrical cavities in an elastic formation. This model was then used 
to assess the influence of receiver position on the propagation of both axisymmetric and non-axisymmetric wave modes when cavities with different cross-sections were examined, namely a circular, an oval, a thin oval, a kidney and a boomerang. Both the time responses and the Fourier spectral representations were included to aid in the visualization of the different wave components.

The amplitude of the guided waves increases as the surface inclusion becomes flatter (greater curvature radius), and then as the side wall becomes concave for receivers placed above the inclusion and in its vicinity. As we move from the circular to the oval and then to the thin oval cross-section cavities, the results indicate progressively slower flexural waves with increasing amplitude, while the pseudo-Rayleigh waves (axisymmetric waves) have higher cutoff frequencies. Therefore, at lower frequencies the dispersions of these two modes reveal different tendencies; but as the frequency increases, their wave velocities converge, and they both denote lower wave velocities, as the ovality ratio increases. These modes lose importance rapidly as the receiver is placed further away from the surface inclusion, and limit their contribution to lower excitation frequencies. The screw mode, however, has a higher cutoff frequency, becomes less dispersive and exhibits lower amplitude, as we move from the circular inclusion to the oval.

The responses obtained when the wall of an oval- or a thin oval-shaped inclusion is deformed (designated here as the kidney- or boomerang-shaped inclusion), are related to those calculated for the former oval, but indicate increasing importance of the higher modes for receivers placed above the cavity and in its vicinity.

The guided waves at a receiver placed on the $x$ axis undergo significant decay, but their contribution is still important. The horizontal displacement results indicate decreasing amplitude as the cavity becomes flatter. The axisymmetric, guided and flexural waves lose significance and their importance is mostly limited to low frequencies. Again, the higher order modes become less important when the ovality of the cavity increases, while their relative importance increases when the side wall of the cavity becomes concave.

\section{Appendix A. The Green's functions}

Definitions

$\begin{array}{ll}\lambda, \mu & \begin{array}{l}\text { Lamé constants } \\ \rho\end{array} \\ \alpha=\sqrt{(\lambda+2 \mu) / \rho} & \text { mass density } \\ \beta=\sqrt{\mu / \rho} & \text { S wave velocity } \\ k_{\mathrm{p}}=\omega / \alpha, k_{\mathrm{s}}=\omega / \beta & \\ k_{\alpha}=\sqrt{k_{\mathrm{p}}^{2}-k_{z}^{2}}, k_{\beta}=\sqrt{k_{\mathrm{s}}^{2}-k_{z}^{2}} \\ A=\frac{1}{4 \mathrm{i} \rho \omega^{2}} & \text { amplitude } \\ \gamma_{i}=\partial r / \partial x_{i}=x_{i} / r, i=1,2 & \text { direction cosines } \\ H_{n \alpha}=H_{n}^{(2)}\left(k_{\alpha} r\right), H_{n \beta}=H_{n}^{(2)}\left(k_{\beta} r\right) \\ B_{n}=k_{\beta}^{n} H_{n \beta}-k_{\alpha}^{n} H_{n \alpha} & \text { Hankel functions }\end{array}$

Green's functions

$$
G_{x x}=A\left[k_{\mathrm{s}}^{2} H_{0 \beta}-\frac{1}{r} B_{1}+\gamma_{x}^{2} B_{2}\right]
$$




$$
\begin{aligned}
& G_{y y}=A\left[k_{\mathrm{s}}^{2} H_{0 \beta}-\frac{1}{r} B_{1}+\gamma_{y}^{2} B_{2}\right] \\
& G_{z z}=A\left[k_{\mathrm{s}}^{2} H_{0 \beta}-k_{z}^{2} B_{0}\right] \\
& G_{x y}=G_{y x}=\gamma_{x} \gamma_{y} A B_{2} \\
& G_{x z}=G_{z x}=\mathrm{i} k_{z} \gamma_{x} A B_{1} \\
& G_{y z}=G_{z y}=\mathrm{i} k_{z} \gamma_{y} A B_{1}
\end{aligned}
$$

Volumetric strain (super-index $=$ direction of load)

$$
\begin{aligned}
\varepsilon_{\mathrm{Vol}}^{l} & =G_{x l, x}+G_{y l, y}+G_{z l, z}=A\left[\frac{\partial}{\partial x_{l}}\left(k_{\mathrm{s}}^{2} H_{0 \beta}\right)+B_{0, x l x}+B_{0, y l y}+B_{0, z l z}\right] \\
& =A \frac{\partial}{\partial x_{l}}\left[k_{\mathrm{s}}^{2} H_{0 \beta}+B_{0, x x}+B_{0, y y}+B_{0, z z}\right]=A \frac{\partial}{\partial x_{l}}\left[k_{\mathrm{s}}^{2} H_{0 \beta}+\hat{\nabla}^{2} B_{0}\right]
\end{aligned}
$$

Note: $H_{0 \beta, l}=-k_{\beta} \gamma_{l} H_{l \beta}, \quad H_{0 \beta, z}=-\mathrm{i} k_{z} H_{0 \beta}$.

Strain components (tensor definition, not engineering)

$$
\begin{aligned}
\varepsilon_{i j}^{l} & =\frac{1}{2}\left(G_{i l, j}+G_{j l, i}\right)=\frac{1}{2} A\left(\delta_{i l} k_{\mathrm{s}}^{2} H_{0 \beta, j}+\delta_{j l} k_{\mathrm{s}}^{2} H_{0 \beta, i}+B_{0, i l j}+B_{0, j l i}\right) \\
& =\frac{1}{2} k_{\mathrm{s}}^{2} A\left(\delta_{i l} H_{0 \beta, j}+\delta_{j l} H_{0 \beta, i}\right)+A B_{0, i j l}
\end{aligned}
$$

Strains for loads in the plane, $l=x, y$

$$
\begin{aligned}
& \varepsilon_{\mathrm{Vol}}^{l}=\gamma_{l} A\left(-k_{\mathrm{s}}^{2} k_{\beta} H_{1 \beta}+k_{z}^{2} B_{1}+\frac{4}{r} B_{2}-B_{3}\right) \\
& \varepsilon_{x x}^{l}=\gamma_{l} A\left(\left(\frac{2}{r} B_{2}-k_{\mathrm{s}}^{2} k_{\beta} H_{1 \beta}\right) \delta_{x l}+\frac{1}{r} B_{2}-\gamma_{x}^{2} B_{3}\right) \\
& \varepsilon_{y y}^{l}=\gamma_{l} A\left(\left(\frac{2}{r} B_{2}-k_{\mathrm{s}}^{2} k_{\beta} H_{1 \beta}\right) \delta_{y l}+\frac{1}{r} B_{2}-\gamma_{y}^{2} B_{3}\right) \\
& \varepsilon_{z z}^{l}=\gamma_{l} k_{z}^{2} A B_{1} \\
& \varepsilon_{x y}^{l}=A\left(\left(\frac{1}{r} B_{2}-\frac{1}{2} k_{\mathrm{s}}^{2} k_{\beta} H_{1 \beta}\right)\left(\delta_{x l} \gamma_{y}+\delta_{y l} \gamma_{x}\right)-\gamma_{x} \gamma_{y} \gamma_{l} B_{3}\right) \\
& \varepsilon_{x z}^{l}=\mathrm{i} k_{z} A\left(\left(\frac{1}{r} B_{1}-\frac{1}{2} k_{\mathrm{s}}^{2} H_{0 \beta}\right) \delta_{x l}-\gamma_{x} \gamma_{l} B_{2}\right) \\
& \varepsilon_{y z}^{l}=\mathrm{i} k_{z} A\left(\left(\frac{1}{r} B_{1}-\frac{1}{2} k_{\mathrm{s}}^{2} H_{0 \beta}\right) \delta_{y l}-\gamma_{y} \gamma_{l} B_{2}\right)
\end{aligned}
$$

Strain for axial loads, $l=z$

$$
\varepsilon_{\mathrm{Vol}}^{z}=\mathrm{i} k_{z} A\left(-k_{\mathrm{s}}^{2} H_{0 \beta}+k_{z}^{2} B_{0}+\frac{2}{r} B_{1}-B_{2}\right)
$$




$$
\begin{aligned}
& \varepsilon_{x x}^{z}=\mathrm{i} k_{z} A\left(\frac{1}{r} B_{1}-\gamma_{x}^{2} B_{2}\right) \\
& \varepsilon_{y y}^{z}=\mathrm{i} k_{z} A\left(\frac{1}{r} B_{1}-\gamma_{y}^{2} B_{2}\right) \\
& \varepsilon_{z z}^{z}=\mathrm{i} k_{z} A\left(-k_{\mathrm{s}}^{2} H_{0 \beta}+k_{z}^{2} B_{0}\right) \\
& \varepsilon_{x y}^{z}=-\mathrm{i} k_{z} \gamma_{x} \gamma_{y} A B_{2} \\
& \varepsilon_{x z}^{z}=\gamma_{x} A\left(-\frac{1}{2} k_{\mathrm{s}}^{2} k_{\beta} H_{1 \beta}+k_{z}^{2} B_{1}\right) \\
& \varepsilon_{y z}^{z}=\gamma_{y} A\left(-\frac{1}{2} k_{\mathrm{s}}^{2} k_{\beta} H_{1 \beta}+k_{z}^{2} B_{1}\right)
\end{aligned}
$$

\section{Stresses}

$$
\tau_{i j}^{l}=\lambda \varepsilon_{\mathrm{Vol}}^{l} \delta_{i j}+2 \mu \varepsilon_{i j}^{l}
$$

\section{References}

[1] M.D. Trifunac, Surface motion of a semi-cylindrical alluvial valley for incident plane SH waves, Bull. Seismol. Soc. Am. 61 (1971) 1755-1770.

[2] H.L. Wong, M.D. Trifunac, Surface motion of semi-elliptical alluvial valley for incident plane SH-waves, Bull. Seismol. Soc. Am. 64 (1974) 1389-1403.

[3] V.W. Lee, On deformations near circular underground cavity subjected to incident plane SH waves, in: Symp. Appl. Comput. Meth. Engrg., University of Southern California, Los Angeles, 1977, pp. 951-961.

[4] S.K. Datta, A.H. Shah, Scattering of SH-waves by embedded cavities, Wave Motion 4 (1982) 265-283.

[5] V.W. Lee, Three-dimensional diffraction of elastic waves by a spherical cavity in an elastic half-space. I. Closed-form solutions, Soil Dyn. Earthquake Engrg. 7 (1988) 149-161.

[6] V.W. Lee, J.A. Karl, Diffraction of SV waves by underground circular, cylindrical cavities, Soil Dyn. Earthquake Engrg. 11 (1992) 445-456.

[7] G. Liu, J. Qu, Guided circumferential waves in a circular annulus, J. Appl. Mech. 65 (1998) $424-430$.

[8] G. Liu, J. Qu, Transient wave propagation in a circular annulus subjected to transient excitation on its outer surface, J. Acoust. Soc. Am. 104 (3) (1998) 1210-1220.

[9] C. Valle, J. Qu, L.J. Jacobs, Guided circumferential waves in layered cylinders, Int. J. Engrg. Sci. 37 (1999) $1369-1387$.

[10] F.J. Sanchez-Sesma, Diffraction of elastic waves by three dimensional surface irregularities, Bull. Seismol. Soc. Am. 73 (1983) 1621-1636.

[11] N. Moeen-Vaziri, M.D. Trifunac, Scattering and diffraction of plane P and SV waves by two-dimensional inhomogeneities: Part II, Soil Dyn. Earthquake Engrg. 7 (1988) 189-200.

[12] V.W. Lee, X. Wu, Application of the weighted residual method to diffraction by 2-D canyons of arbitrary shape. II. Incident P, SV and Rayleigh waves, Soil Dyn. Earthquake Engrg. 13 (1994) 365-375.

[13] G. Waas, Linear two-dimensional analysis of soil dynamics problems in semi-infinite layered media, Ph.D. dissertation, University of California, Berkeley, CA, 1972.

[14] J. Lysmer, T. Udaka, H.B. Seed, R. Hwang, LUSH-A computer program for complex response analysis of soil-structure systems, Report No. EERC 74-4, Earthquake Engineering Research Center, 1974, University of California, Berkeley, CA, 1974.

[15] E. Kausel, Forced vibrations of circular foundations in layered media, MIT Research Report 70-3, Department of Civil Engineering, Massachusetts Institute of Technology, Cambridge, MA, 1974.

[16] A. Ohtsuki, K. Harumi, Effect of topography and subsurface inhomogeneities on seismic SV waves, Earthquake Engrg. Struct. Dyn. 11 (1983) 441-462.

[17] G.D. Manolis, D.E. Beskos, Dynamic response of lined tunnels by an isoparametric boundary element method, Comput. Meth. Appl. Mech. Engrg. 36 (1983) 291-307. 
[18] S.V. Tsinopoulos, S.E. Kattis, D. Polyzos, D.E. Beskos, An advanced boundary element method for axisymmetric elastodynamic analysis, Comput. Meth. Appl. Mech. Engrg. 175 (1999) 53-70.

[19] A.H. Shah, K.C. Wong, S.K. Datta, Diffraction of plane SH waves in a half-space, Earthquake Engrg. Struct. Dyn. 10 (1982) $519-528$.

[20] H.A. Pedersen, F.J. Sánchez-Sesma, M. Campillo, Three-dimensional scattering by two-dimensional topographies, Bull. Seismol. Soc. Am. 84 (1994) 1169-1183.

[21] J.E. Luco, F.C.P. De Barros, Seismic response of a cylindrical shell embedded in a layered viscoelastic half-space. I. Formulation, Earthquake Engrg. Struct. Dyn. 23 (5) (1994) 553-567.

[22] F.C.P. De Barros, J.E. Luco, Seismic response of a cylindrical shell embedded in a layered viscoelastic half-space. II. Validation and numerical results, Earthquake Engrg. Struct. Dyn. 23 (5) (1994) 569-580.

[23] A.A. Stamos, D.E. Beskos, 3-D seismic response analysis of long lined tunnels in half-space, J. Soil Dyn. Earthquake Engrg. 15 (1996) 111-118.

[24] L. Zhang, A.K. Chopra, Three-dimensional analysis of spatially varying ground motions around a uniform canyon in a homogeneous half-space, Earthquake Engrg. Struct. Dyn. 20 (10) (1991) 911-926.

[25] L. Zhang, A.K. Chopra, Impedance functions for three-dimensional foundations supported on an infinitely-long canyon of uniform cross-section in a homogeneous half-space, Earthquake Engrg. Struct. Dyn. 20 (11) (1991) 1011-1027.

[26] J.S. Bell, D.I. Gough, Northeast-southwest compressive stress in Alberta-evidence from oil wells, Earth Planet. Sci. Lett. 45 (1979) 475-482.

[27] Z. Zheng, K. Kemeny, N.G.W. Cook, Analysis of borehole breakouts, J. Geophy. Res. 94 (1989) 171-182.

[28] A.D. Burden, The propagation of elastic surface waves along cylindrical cavities of general cross section, Wave Motion 7 (1985) $153-168$.

[29] A. Bamberger, P. Joly, M. Kern, Propagation of elastic surface waves along a cylindrical cavity of arbitrary cross section, Math. Modelling Numer. Anal. 25 (1) (1991) 1-30.

[30] M. Bouchon, K. Aki, Discrete wave-number representation of seismic-source wave field, Bull. Seismol. Soc. Am. 67 (1977) 259-277.

[31] R.A. Phinney, Theoretical calculation of the spectrum of first arrivals in layered elastic mediums, J. Geophys. Res. 70 (1965) 5107-5123.

[32] G.D. Manolis, D.E. Beskos, Boundary Element Methods in Elastodynamics, Unwin Hyman, London, 1988.

[33] P.K. Banerjee, The Boundary Element Methods in Engineering, McGraw-Hill, New York, 1994.

[34] C.A. Brebbia, J.C. Telles, L.C. Wrobel, Boundary Elements Techniques. Theory and Applications in Engineering, Springer, Berlin, 1984.

[35] A.J.B. Tadeu, P.F.A. Santos, E. Kausel, Closed-form integration of singular terms for constant, linear and quadratic boundary elements. Part I: SH wave propagation, EABE_Engrg. Anal. Bound. Elem. 23 (8) (1999) 671-681.

[36] A.J.B. Tadeu, P.F.A. Santos, E. Kausel, Closed-form integration of singular terms for constant, linear and quadratic boundary elements. Part II: SV-P wave propagation, EABE—Engrg. Anal. Bound. Elem. 23 (9) (1999) 757-768.

[37] Y.H. Pao, C.C. Mow, Diffraction of Elastic Waves and Dynamic Stress Concentrations, Crane and Russak, New York, 1973.

[38] E. Kausel, J.M. Roesset, Frequency domain analysis of undamped systems, J. Engrg. Mech., ASCE 118 (4) (1992) $721-734$.

[39] W.M. Ewing, W.S. Jardetzky, F. Press, Elastic Waves in Layered Media, McGraw-Hill, New York, 1957. 